\title{
CONTROL DE Prostephanus truncatus (Horn) EN SEMILLA DE MAÍZ ALMACENADA 1
}

\author{
Abel Valdes 2 , Elos Mariano Mendoza ${ }^{2}$,Francisco Nieto ${ }^{2}$
}

\begin{abstract}
RESUMEN
Control de Prostephanus truncatus (Horn) en semilla de maíz almacenada. Se evaluó la efectividad biológica de nueve productos insecticidas solos y en mezcla, empleados para el control de plagas del maíz (granos almacenados y del cultivo) y a dosis recomendada para este cultivo. Se aplicaron por inmersión del grano sobre una variedad de maíz tuxpeño para observar el efecto biológico en términos de mortalidad sobre el gorgojo mayor de los granos Prostephanus truncatus, durante un período de almacenamiento de seis meses, bajo un diseño completamente al azar con 38 tratamientos, tres repeticiones y una comparación de medias (Tukey 0,05); con el objeto de analizar el insecticida que presente la mejor opción para el tratamiento a la semilla de maíz almacenada para investigación. El análisis de varianza indicó diferencias significativas en porcentaje de mortalidad de los insectos para cada uno de los tratamientos. El efecto de los insecticidas fue de $100 \%$ a $5.53 \%$ de mortalidad. Los insecticidas deltametrina, permetrina y carbaryl expresaron mayor porcentaje de mortalidad sobre los demás, incluyendo el testigo (0 \% de mortalidad). Las mezclas en las que se tuvo buen resultado son: deltametrina + pirimifos metil, deltametrina + carbaryl, permetrina + carbaryl, carbaryl + pirimifos metil, carbaryl + clorpirifos etil. La prueba de germinación de semillas al final de la evaluación mostró, que la viabilidad de la misma no fue afectada por los tratamientos.
\end{abstract}

\begin{abstract}
Prostephanus truncatus control in maize's seed. The objective considers to evaluate the biological effectiveness of 9 single insecticide products and in mixture, employees for the control of plagues of the maize (grain stored and of the culture) and to dose recommended for this culture. They were applied by immersion of the grain on a variety of maize tuxpeño to observe the biological effect in mortality terms on large grain borer Prostephanus truncatus, during a period of storage of 6 months, under a design completely at random with 38 treatments, 3 repetitions and one comparison of averages (Tukey 0,05 ); with the intention of analyzing the insecticide that presents the best option for the treatment to the maize seed stored for investigation. The variance analysis indicated significant differences in percentage of mortality of the insects for each one of the treatments. The effect insecticides was of $100 \%$ to $5.53 \%$ of mortality. The insecticides deltametrina, permetrina and carbaryl expressed greater percentage of mortality on the others, including the witness ( $0 \%$ mortality). The mixtures in which good result was had are: deltametrina + pirimifos metil, deltametrina + carbaryl, permetrina + carbaryl, carbaryl + pirimifos metil, carbaryl + clorpirifos etil. The test of germination of seeds at the end of the evaluation showed, that the viability of the same one was not affected by the treatments.
\end{abstract}

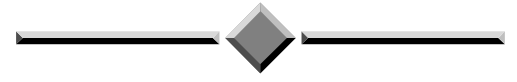

\section{INTRODUCCIÓN}

Considerando al maíz como el principal producto de subsistencia en el área rural de México, los agricultores tienen la necesidad de almacenar la semilla que seleccionan para sembrar de un ciclo a otro y para combatir plagas de granos almacenados invierten gran cantidad de dinero en fumigantes e insecticidas. Muchas veces estos gastos son inútiles y aumentan los costos de almacenaje y no aportan los beneficios esperados, debido al mal uso o a la baja calidad de los productos aplicados, así como a la resistencia adquirida por los insectos.

Se estima que el $5 \%$ de todos los granos cosechados se pierden antes de su consumo. Estas pérdidas varía en cada país y cada año. En México, se pierde hasta un $25 \%$ de la producción total de maíz, trigo y frijol, especialmente en áreas bajas, cálidas y húmedas del país por ser condiciones ecológicas adecuadas para la infestación por insectos, roedores, ácaros, hongos y

\footnotetext{
1 Presentado en la XLV Reunión Anual del PCCMA, Guatemala, 1999.

2 Universidad Autónoma Agraria “Antonio Narro”. Buenavista, Saltillo, Coah, Méx. E mail: abel_mx@yahoo. com
} 
otros microorganismos. Siendo aproximadamente unas 100 especies de insectos en el mundo las responsables de daños a alimentos almacenados, existiendo en México algo más de 25 especies pertenecientes a los Ordenes Coleoptera y Lepidoptera; de éstas, alrededor de 15 son las de mayor importancia (Ramírez et al; 1980).

Conocido comúnmente como gorgojo barrenador de los granos, es un insecto que ataca vorazmente a todos los cereales y sus productos. Se reproducen y desarrollan sobre el frijol almacenado pero no lo dañan (Ramayo, 1983). Los adultos y las larvas originan perforaciones en los granos; se alimentan del endospermo y producen polvo abundante; comen el interior del grano dejando la cubierta (Ramírez, 1978).

\section{Métodos de Combate.}

Los métodos de prevención y sanidad son llamados combate indirecto, consistiendo en algunas reglas generales para la limpieza y almacenamiento del grano y cuando se ejerce la destrucción de plagas mediante procedimientos químicos, físicos, mecánicos y biológicos, resulta el combate directo, teniendo cada uno de éstos sus ventajas y desventajas. Para utilizar cualesquiera de estos métodos es necesario conocer una serie de factores biológicos, ecológicos, etológicos, fisiológicos y económicos (Ramírez et al; 1980).

La CICOPLAFEST (1994), menciona que los productos autorizados para su aplicación en granos almacenados son: clorpirifos metil, deltametrina, diclorvos (naled), fenitrotion, foxim, malatión, pirimifos metil y los fumigantes bromuro de metilo, fosfuro de aluminio $\mathrm{y}$ fosfuro de magnesio.

(Rees, 1991). Menciona que Teretriosoma nigrescens Lewis (Coleóptera: Histeridae) es buen depredador de P. truncatus (Horn) en México y Centro América, pero no en Africa. En México (Bañuelos, 1992) demostró que Teretriosoma nigrescens Lewis presentó actividad depredadora sobre todos los estados de desarrollo de $P$. truncatus (Horn) con preferencia sobre los dos primeros instares larvales.

La exposición al sol es un método en el que los insectos abandonan al grano que es expuesto; sin embargo, el asoleado no siempre mata huevecillos y larvas que permanecen en el interior del grano. El ahumado del grano lo utilizan algunos agricultores que almacenan su semilla sin desgranar en plataformas elevadas de madera y hacen pasar humo por debajo de éstas. Otros almacenan el grano cosechado en el techo de sus casas o del lugar que utilizan para cocinar. El humo y el calor del fuego ahuyentan los insectos del grano, además de mantener el grano seco y lo protege de una nueva inva- sión de insectos. Otra alternativa para el almacenamiento de granos consiste en colocar el grano en recipientes herméticos, por lo que los insectos mueren por falta de suficiente oxígeno. En algunos lugares, los agricultores almacenan el grano en depósitos subterráneos y muy secos que pueden estar completamente herméticos. Otros tipos de recipientes herméticos pueden ser más difíciles de construir y de mantener en buenas condiciones (Lindblad y Druben, 1986).

Mensah and White (1984) recomiendan usar polvos minerales y tierra de diatomeas impregnadas con malathión para dar un control efectivo contra gorgojos. Mas tarde Sánchez (1987), demostró que los tratamientos de polvos minerales por sí mismos como: tezontle rojo, tezontle negro, cal, carbonato de calcio, ceniza del chinchonal, primex, salsorcite, teckies ligero, teckies pesado, mármol y rob son relativamente efectivos para controlar a $P$. truncatus.

En muchos lugares se mezclan plantas locales con el grano, pero ya que las plantas difieren de una a otra parte del mundo. Es necesario estudiar con mayor detalle estos métodos naturales de control o cualquier otro que de resultados positivos en el control de insectos de almacén sin utilizar insecticidas (Lindblad y Druben, 1986). El polvo del vegetal Ricinus. communis (Euphorbiaceae) conocido como higuerilla, en su dosis al $1 \%$, es recomendable para usarlo como protector del grano sin riesgo a la salud (Aguilera, 1991).

La forma más fácil para complementar un combate de plagas está dada por métodos de limpieza e inspección, aunados a procedimientos de combate químico, mecánico, cultural o legal (Ramírez, 1978).

El uso de feromonas es una diferente técnica moderna en el control de insectos de productos almacenados. P. truncatus responde a dominicalure 2. 1-methylbutyl (E)-2,4-dímethyl-2-pentenoate (dominicalure 2) sobre el macho. El uso principal de las feromonas es el de realizar monitoreos y posteriormente aplicar un método de control (Burkholder, 1985).

Sin embargo un factor limitante de importancia para el uso de insecticidas en el combate de las plagas que atacan a los granos almacenados es el empleo o destino final que se dará al grano. El tratamiento de los granos almacenados con sustancias químicas tiene dos puntos de gran importancia que deben analizarse con cuidado:

1. Granos que serán empleados como simiente y en cuyo caso los insecticidas utilizados no deben dañar el poder germinativo del mismo, o en caso de daño, éste debe ser mínimo y nunca superior de un $5 \%$. 
2. Granos que serán utilizados en la elaboración de productos destinados al consumo humano y de animales domésticos y en cuyo caso los compuestos químicos utilizados no deben ser tóxicos a los animales de sangre caliente.

El tratamiento de semillas con protectores químicos es el medio más eficaz para lograr una protección contra organismos patógenos, insectos y roedores, que son los que ocasionan problemas de germinación y vigor de semillas. El medio de aplicar el tratamiento de semilla puede ser en polvo seco, solución acuosa, líquido, aspersión y neblina (Facio, 1983). La aplicación del tratamiento en las formas descritas anteriormente se realizan con equipos especiales (tratadoras) disponibles para la aplicación de cada tratamiento.

En lo referente a control por fumigantes, el término fumigante, incluye a todos aquellos materiales que ejercen su acción tóxica en estado gaseoso. La principal ventaja es su penetración, ya que estos materiales se introducen en todas partes de espacio disponible; su desventaja es que sus vapores se dispersan con rapidez, siendo por lo tanto, apropiados solamente para espacios cerrados. No son materiales adecuados para ejercer efecto residual. Causan la muerte de los organismos interfiriendo en una u otra forma la asimilación del oxígeno por los tejidos. Martínez y Velazco (1982), demostraron que almacenar maíz desgranado y fumigado con phostoxin en tambores, nulifica casi en forma completa la infestación de insectos y su daño.

Un factor muy importante de pérdidas causado a la semilla de investigación que es almacenada, es el daño por los insectos concretamente Prostephanus truncatus. Esto genera en sí pérdidas económicas considerables, tanto en bodegas como en centros de investigación para mejoramiento genético; y por su valioso contenido es necesario que no sufra daño de ningún tipo. Por lo anteriormente expuesto el objetivo del presente trabajo es el de evaluar la efectividad biológica de nueve insecticidas solos y en mezcla en semilla tratada, empleados para el control de Prostephanus truncatus durante un período de almacenamiento de seis meses.

\section{MATERIALES Y MÉTODOS}

El presente trabajo se realizó en el departamento de Parasitología de la Universidad Autónoma Agraria “Antonio Narro" en Buenavista, Saltillo, Coah; México, en el año de 1997. Antes de iniciar esta investigación se hicieron ensayos preliminares para observar el comportamiento de los insectos en el grano de maíz, determinar los productos y dósis a usar y para escoger el diseño experimental que más se ajustara a la realización del trabajo.

Para tener suficientes insectos se formó una colonia con un tipo de grano de una variedad de textura blanda, susceptible al ataque de los insectos usados en el experimento, para facilitar el desarrollo de los mismos, se utilizó la variedad de maíz Tuxpeño, cultivado en el estado de Guanajuato.

Este trabajo consistió en evaluar la efectividad biológica de ocho productos insecticidas y cal comercial sobre P. truncatus, tanto solos como mezclados (excepto la cal), por lo que se incluyeron los 37 tratamientos mostrados en el Cuadro 1.

Cuadro 1. Tratamiento a la semilla de maíz para el control de P. truncatus. México, 1997.

\begin{tabular}{|c|c|c|}
\hline $\operatorname{Tra}^{1}$ & Insecticidas & $\begin{array}{l}\text { Dosis: } \\
\text { Ce ó gr } / 2 \text { litros } \\
\text { de agua }\end{array}$ \\
\hline 1 & pirimifos metil $50 \mathrm{CEa}$ & $8 \mathrm{ml}$. \\
\hline 2 & deltametrina $25 \mathrm{CE}$ & $3.33 \mathrm{ml}$. \\
\hline 3 & clorpirifos etil $48 \mathrm{CE}$ & $4.1 \mathrm{ml}$. \\
\hline 4 & paratión metílico $50 \mathrm{CE}$ & $5 \mathrm{ml}$. \\
\hline 5 & permetrina $50 \mathrm{CE}$ & $1.7 \mathrm{ml}$. \\
\hline 6 & clorpirifos metil $48 \mathrm{CE}$ & $2.5 \mathrm{ml}$. \\
\hline 7 & carbaryl $80 \mathrm{PHb}$ & 10 gr. \\
\hline 8 & endosulfán $38 \mathrm{CE}$ & $20 \mathrm{ml}$. \\
\hline 9 & cal comercial & 200 gr. \\
\hline 10 & pirimifos metil $50 \mathrm{CE} /$ deltametrina $25 \mathrm{CE}$ & $4 \mathrm{ml} . / 1.6 \mathrm{ml}$. \\
\hline 11 & pirimifos metil $50 \mathrm{CE} /$ clorpirifos etil $48 \mathrm{CE}$ & $4 \mathrm{ml} . / 2.05 \mathrm{ml}$ \\
\hline 12 & pirimifos metil $50 \mathrm{CE}$ /paratión metílico $50 \mathrm{CE}$ & $4 \mathrm{ml} . / 2.5 \mathrm{ml}$ \\
\hline 13 & pirimifos metil $50 \mathrm{CE} /$ permetrina $50 \mathrm{CE}$ & $4 \mathrm{ml} . / 0.85 \mathrm{ml}$ \\
\hline 14 & pirimifos metil $50 \mathrm{CE} /$ clorpirifos metil $48 \mathrm{CE}$ & $4 \mathrm{ml} . / 1.125 \mathrm{ml}$. \\
\hline 15 & pirimifos metil $50 \mathrm{CE} /$ carbaryl $80 \mathrm{PH}$ & $4 \mathrm{ml} . / 5 \mathrm{gr}$. \\
\hline 16 & pirimifos metil $50 \mathrm{CE} /$ endosulfán $38 \mathrm{CE}$ & $4 \mathrm{ml} . / 10 \mathrm{ml}$. \\
\hline 17 & deltametrina $25 \mathrm{CE} /$ clorpirifos etil $48 \mathrm{CE}$ & $1.6 \mathrm{ml} . / 2.05 \mathrm{ml}$. \\
\hline 18 & deltametrina $25 \mathrm{CE} /$ paratión metílico $50 \mathrm{CE}$ & $1.6 \mathrm{ml} . / 2.5 \mathrm{ml}$. \\
\hline 19 & deltametrina $25 \mathrm{CE} /$ permetrina $50 \mathrm{CE}$ & $1.6 \mathrm{ml} . / 0.85 \mathrm{ml}$. \\
\hline 20 & deltametrina $25 \mathrm{CE} /$ clorpirifos metil $48 \mathrm{CE}$ & $1.6 \mathrm{ml} . / 1.125 \mathrm{ml}$. \\
\hline 21 & deltametrina $25 \mathrm{CE} /$ carbaryl $80 \mathrm{PH}$ & $1.6 \mathrm{ml} . / 5 \mathrm{gr}$. \\
\hline 22 & deltametrina $25 \mathrm{CE} /$ endosulfán $38 \mathrm{CE}$ & $1.6 \mathrm{ml} . / 10 \mathrm{ml}$ \\
\hline 23 & clorpirifos etil $48 \mathrm{CE} /$ paratión metílico $50 \mathrm{CE}$ & $2.05 \mathrm{ml} . / 2.5 \mathrm{ml}$. \\
\hline 24 & clorpirifos etil $48 \mathrm{CE} /$ permetrina $50 \mathrm{CE}$ & $2.05 \mathrm{ml} . / 0.85 \mathrm{ml}$. \\
\hline 25 & clorpirifos etil $48 \mathrm{CE} /$ clorpirifos metil $48 \mathrm{CE}$ & $2.05 \mathrm{ml} . / 1.125 \mathrm{ml}$. \\
\hline 26 & clorpirifos etil $48 \mathrm{CE} /$ carbaryl $80 \mathrm{PH}$ & $2.05 \mathrm{ml} . / 5 \mathrm{gr}$. \\
\hline 27 & clorpirifos etil $48 \mathrm{CE} /$ endosulfán $38 \mathrm{CE}$ & $2.05 \mathrm{ml} . / 10 \mathrm{ml}$. \\
\hline 28 & paratión metílico $50 \mathrm{CE} /$ permetrina $50 \mathrm{CE}$ & $2.5 \mathrm{ml} . / 0.85 \mathrm{ml}$ \\
\hline 29 & paratión metílico $50 \mathrm{CE} /$ clorpirifos metil $48 \mathrm{CE}$ & $2.5 \mathrm{ml} . / 1.125 \mathrm{ml}$. \\
\hline 30 & paratión metílico $50 \mathrm{CE} /$ carbaryl $80 \mathrm{PH}$ & $2.5 \mathrm{ml} . / 5 \mathrm{gr}$. \\
\hline 31 & paratión metílico $50 \mathrm{CE}$ /endosulfán $38 \mathrm{CE}$ & $2.5 \mathrm{ml} . / 10 \mathrm{ml}$. \\
\hline 32 & permetrina $50 \mathrm{CE} /$ clorpirifos metil $48 \mathrm{CE}$ & $0.85 \mathrm{ml} . / 1.125 \mathrm{ml}$. \\
\hline 33 & permetrina $50 \mathrm{CE} /$ carbaryl $80 \mathrm{PH}$ & $0.85 \mathrm{ml} . / 5 \mathrm{gr}$. \\
\hline 34 & permetrina $50 \mathrm{CE} /$ endosulfán $38 \mathrm{CE}$ & $0.85 \mathrm{ml} . / 10 \mathrm{ml}$ \\
\hline 35 & clorpirifos metil $48 \mathrm{CE} /$ carbaryl $80 \mathrm{PH}$ & $1.125 \mathrm{ml} . / 5 \mathrm{gr}$. \\
\hline 36 & clorpirifo metil/endosulfán $38 \mathrm{CE}$ & $1.125 \mathrm{ml} . / 10 \mathrm{ml}$. \\
\hline 37 & carbaryl $80 \mathrm{PH} /$ endosulfán $38 \mathrm{CE}$ & $5 \mathrm{gr} . / 10 \mathrm{ml}$ \\
\hline
\end{tabular}

Tra ${ }^{1}=$ Número de tratamiento.

$\mathrm{CEa}=$ Concentrado emulsionable

$\mathrm{PHb}=$ Polvo humectable 


\section{Insecticidas utilizados en el estudio}

Siguiendo las recomendaciones de González (1988), se utilizaron los insecticidas, adherente Agral plus y un colorante artificial en dosis de $1 \mathrm{ml} . / 2 \mathrm{l}$. de agua y $2 \mathrm{~g} / 21$ de agua respectivamente (Cuadro 1).

\section{Descripción de los insecticidas utilizados (CICOPLAFEST,1994).}

Carbaryl es un plaguicida: insecticida carbámico de contacto e ingestión. En el mercado se puede localizar en la presentación granulado al 50\%; polvo al 50, 75 y $100 \%$; polvo humectable al $80 \%$ y suspensión acuosa al 30 y $48 \%$.

Clorpirifos metil, plaguicida: insecticida organofosforado de contacto. Presentación: polvo al 30\% utilizada para tratamiento de granos para consumo humano y concentrado emulsionable al $48 \%$ para tratamiento previo de almacenes y transporte.

Clorpirifos etil, insecticida organofosforado de contacto. Presentación: concentrado emulsionable 480.00 g. i.a./Kg. ó L. y granulado 20.00 y 30.00 g. i.a./kg. ó L.

Deltametrina, insecticida piretroide de contacto. Presentación: concentrado emulsionable al $25 \%$ utilizado para aplicación al follaje y tratamiento de granos almacenados para consumo humano.

Endosulfán, insecticida acaricida, éster del ácido sulfuroso de un diol cíclico clorado. Presentación: concentrado emulsionable al 37,5, 37,8 y $38 \%$. Polvo humectable al 37,8 y $40 \%$

Paratión metilico, insecticida organofosforado de contacto e ingestión. Presentación: concentrado emulsionable al 50, 52.5, 53.6, 54 y $72 \%$. En polvo al 20 y $30 \%$ y en suspensión acuosa al $24 \%$.

Permetrina, insecticida piretroide de contacto. Presentación: concentrado emulsionable al 34 y $50 \%$. Granulado al $4 \%$.

Pirimifos metil, insecticida organofosforado de contacto. Presentación: tratamiento de semilla para siembra: Polvo 20\%. Control de plagas en ornamentales, instalaciones de almacenamiento y tratamiento de granos y semillas almacenadas: concentrado emulsionable al $50 \%$.

La manipulación de los insectos se realizó con cribas, separando de esta forma grano entero, partido, insectos y harina; metodología empleada por Wright et al (1987).

Se trabajó con maíz tuxpeño y $P$. truncatus en 38 tratamientos con tres repeticiones, tomando el dato de mortalidad a las 72 horas después de la exposición. Para detectar las diferencias entre tratamientos se utilizó un diseño completamente al azar y una comparación de medias con una prueba de Tukey al 0,05. Además se aplicó la siguiente fórmula para corregir la mortalidad de los tratamientos respecto al testigo.

$$
\begin{array}{cc}
\mathrm{MC}=\mathrm{X}-\mathrm{Y} & 100 \\
\mathrm{X} &
\end{array}
$$

Donde:

$\mathrm{MC}=$ Mortalidad Corregida

$\mathrm{X}=\%$ de sobrevivientes en el testigo.

$\mathrm{Y}=\%$ de sobrevivientes en el tratamiento.

El tratamiento de los $250 \mathrm{~g}$ del grano de maíz se realizó aplicando el insecticida en cada bolsa de polietileno, posteriormente se procedió a sumergir el grano agitando durante 10 segundos de tal manera que el insecticida se distribuyera uniformemente sobre la superficie del grano formando una película protectora, el grano se seco por exposición al sol. Los granos tratados se mantuvieron en condiciones similares a una bodega, sin controlar humedad y temperatura. La humedad del grano después de tratarlo estuvo entre un 12 y $13 \%$.

Después de estar el grano almacenado por seis meses se infestaron las muestras de $250 \mathrm{~g}$ de semilla tratada, con 30 insectos vigorosos; a las 72 horas siguientes se hizo el conteo de mortalidad, retirando insectos vivos y muertos, así como grano dañado (Garrido, 1970).

Se utilizaron frascos de 0.51 . de capacidad, se tomó una muestra de $250 \mathrm{~g}$ de semilla por frasco y se emplearon 38 frascos para cada repetición; incluyendo un testigo para cada repetición.

Para evaluar el efecto de los productos empleados para proteger los granos se hicieron las siguientes observaciones:

a) Mortalidad.- A las 72 horas siguientes de la infestación se cribó el grano, separando los insectos vivos y muertos, calculándose los datos en porcentaje de mortalidad.

b) Poder residual.-La prueba de mortalidad realizada seis meses después del tratamiento de la semilla permitió evaluar la efectividad de cada producto y determinar el poder residual en base a mortalidad observada por conteo en las muestras. 
c) Viabilidad de las semillas.- Se realizó una prueba de germinación para cada tratamiento al final de los seis meses. Con anterioridad se hizo una prueba de germinación inicial.

\section{RESULTADOS Y DISCUSIÓN}

Los resultados del presente estudio se presentan con dos apartados, el efecto de los tratamientos sobre $P$. truncatus y sobre la germinación de la semilla.

\section{Efecto de los tratamientos sobre $P$. truncatus.}

En la prueba realizada, existió diferencia para el porciento de mortalidad de los insectos en cada uno de los tratamientos. Se tomaron como mejores los tratamientos que presentaron un porcentaje de mortalidad del $100 \%$. El análisis de varianza (Cuadro 2) muestra alta significancia al nivel de $1 \%$ para la fuente de variación tratamientos, indicando que el porciento de mortalidad provocado por cada tratamiento es diferente. Los resultados obtenidos son confiables, respaldado esto por el valor adecuado del coeficiente de variación de $12,11 \%$. La prueba de Tukey con un valor de 25.2866 permitió agrupar a los tratamientos en diferentes grupos estadísticos (Cuadro 3), lo cual permite seleccionar un insecticida, en base a otras características.

De acuerdo a los resultados del Cuadro 3 los insecticidas permetrina, deltametrina y carbaryl presentan el $100 \%$ de Mortalidad, mientras que las mezclas pirimifos metil + deltametrina, clorpirifos etil + carbaryl, permetrina + carbaryl, pirimifos metil + carbaryl, deltametrina + carbaryl, tuvieron también el $100 \%$ de mortalidad.

En este mismo Cuadro 3 se observa que los insecticidas y mezclas que tuvieron buenos resultados como son: los insecticidas solos deltametrina, permetrina y carbaryl; combinaron bien en las mezclas con todos los demás. Aunque algunos insecticidas en mezcla no funcionaron en un $100 \%$, estadísticamente son iguales

Cuadro 2. Análisis de Varianza de los porcentajes de mortalidad en $P$. truncatusobtenidos a los seis meses de almacenamiento de maíz. México, 1997.

\begin{tabular}{lrrrrr}
\hline FV & GL & \multicolumn{1}{c}{ SC } & CM & F & P>F \\
\hline TRAT. & 37 & 66348.25 & 1793.20 & $25.3812 * *$ & 0.00 \\
ERROR & 76 & 5369.44 & 70.65 & & \\
TOTAL & 113 & 71717.69 & & & \\
C.V.=12.11\% & & & & \\
\hline
\end{tabular}

**Significativo al $1 \%$ de probabilidad.
Cuadro 3. Prueba de comparación de medias por Tukey al $0,05 \%$ de los porcentajes de mortalidad sobre $P$. truncatus de 37 tratamientos efectuados en maíz. México, 1997.

\begin{tabular}{|c|c|c|c|}
\hline $\operatorname{Tr}$ & Insecticidas & $\begin{array}{l}\% \\
\text { M. }\end{array}$ & $\begin{array}{c}\text { Tukey } \\
\mathbf{0 , 0 5}\end{array}$ \\
\hline 2 & Deltametrina & 100 & $A^{*}$ \\
\hline 5 & permetrina & 100 & A \\
\hline 7 & Carbaryl & 100 & A \\
\hline 10 & pirimifos metil + deltametrina & 100 & A \\
\hline 15 & pirimifos metil + carbaryl & 100 & A \\
\hline 21 & deltametrina + carbaryl & 100 & A \\
\hline 26 & clorpirifos etil + carbaryl & 100 & A \\
\hline 33 & permetrina + carbaryl & 100 & A \\
\hline 13 & pirimifos metil + permetrina & 98.86 & $\mathrm{AB}$ \\
\hline 19 & deltametrina + permetrina & 98.86 & $\mathrm{AB}$ \\
\hline 28 & paratión metílico + permetrina & 98.86 & $\mathrm{AB}$ \\
\hline 30 & paratión metílico + carbaryl & 98.86 & $\mathrm{AB}$ \\
\hline 32 & permetrina + clorpirifos metil & 98.86 & $\mathrm{AB}$ \\
\hline 34 & permetrina + endosulfán & 98.86 & $\mathrm{AB}$ \\
\hline 35 & clorpirifos metil + carbaryl & 98.86 & $\mathrm{AB}$ \\
\hline 37 & carbaryl + endosulfán & 98.86 & $\mathrm{AB}$ \\
\hline 18 & deltametrina + paratión metílico & 97.76 & $\mathrm{ABC}$ \\
\hline 22 & deltametrina + endosulfán & 97.76 & $\mathrm{ABC}$ \\
\hline 24 & clorpirifos etil + permetrina & 96.66 & $\mathrm{ABC}$ \\
\hline 20 & deltametrina + clorpirifos metil & 95.53 & $\mathrm{ABC}$ \\
\hline 16 & pirimifos metil + endosulfán & 85.53 & $\mathrm{ABCD}$ \\
\hline 17 & deltametrina + clorpirifos etil & 85.53 & $\mathrm{ABCD}$ \\
\hline 27 & clorpirifos etil + endosulfán & 84.43 & $\mathrm{ABCD}$ \\
\hline 36 & clorpirifos metil + endosulfán & 84.43 & $\mathrm{ABCD}$ \\
\hline 31 & paratión metílico + endosulfán & 80 & $\mathrm{ABCD}$ \\
\hline 1 & pirimifos metil & 78.86 & BCDE \\
\hline 4 & paratión metílico & 78.86 & BCDE \\
\hline 11 & pirimifos metil + clorpirifos etil & 78.86 & $\mathrm{BCDE}$ \\
\hline 8 & Endosulfán & 76.66 & BCDE \\
\hline 14 & pirimifos metil + clorpirifos metil & 75.53 & CDE \\
\hline 3 & clorpirifos etil & 61.1 & $\mathrm{DEF}$ \\
\hline 12 & pirimifos metil + paratión metílico & 60 & $\mathrm{DEF}$ \\
\hline 23 & clorpirifos etil + paratión metílico & 37.76 & EFG \\
\hline 25 & clorpirifos etil + clorpirifos metil & 32.2 & FG \\
\hline 29 & paratión metílico + clorpirifos metil & 25.53 & $\mathrm{GH}$ \\
\hline 6 & clorpirifos metil & 17.76 & $\mathrm{GH}$ \\
\hline \multirow[t]{3}{*}{9} & cal comercial & 5.53 & $\mathrm{H}$ \\
\hline & $\mathrm{Tr}=$ Número de tratamiento. & & \\
\hline & $\% \mathrm{M} .=$ Porcentaje de mortalidad. & \multicolumn{2}{|r|}{ Tukey $=25.2866$} \\
\hline
\end{tabular}

*Tratamientos con la misma letra son estadísticamente iguales.

a las que causaron un $100 \%$ de mortalidad, en base al valor de tukey $=25.2866$, esto permite seleccionar entre los insecticidas aquel cuyo costo sea menor, su capacidad toxicológica sobre animales de sangre caliente sea menor, que al realizar el tratamiento sea de olor menos desagradable al personal y sobre todo poder hacer una rotación de insecticidas para reducir el riesgo de provocar resistencia de los insectos a los insecticidas. De acuerdo al valor de Tukey $(0,05)$, existen relaciones positivas entre las mezclas de los insecticidas: deltametrina + pirimifos metil, deltametrina + clorpirifos metil, deltametrina + clorpirifos etil, deltametrina + permetrina, deltametrina + carbaryl, deltametrina + paratión 
Cuadro 4. Porcentajes de germinación de la semilla, obtenidos de los 37 tratamientos y un testigo seis meses después del tratamiento con insecticida en maíz. México, 1997.

\begin{tabular}{|c|c|c|c|}
\hline $\begin{array}{l}\text { No. } \\
\text { Trat. }\end{array}$ & Tratamiento & $\%$ Ger. & $\begin{array}{l}\text { \% Red. } \\
\text { Germ. }\end{array}$ \\
\hline 28 & paratión metílico + permetrina & 87.50 & 10.50 \\
\hline 31 & paratión metílico + endosulfán & 87.50 & 10.50 \\
\hline 35 & clorpirifos metil + carbaryl & 87.50 & 10.50 \\
\hline 27 & clorpirifos etil + endosulfán & 88.75 & 9.25 \\
\hline 29 & paratión metílico + clorpirifos metil & 88.75 & 9.25 \\
\hline 30 & paratión metílico + carbaryl & 88.75 & 9.25 \\
\hline 32 & permetrina + clorpirifos metil & 88.75 & 9.25 \\
\hline 33 & permetrina + carbaryl & 88.75 & 9.25 \\
\hline 36 & clorpirifos metil + endosulfán & 88.75 & 9.25 \\
\hline 37 & carbaryl + endosulfán & 88.75 & 9.25 \\
\hline 20 & deltametrina + clorpirifos metil & 90.00 & 8.00 \\
\hline 34 & permetrina + endosulfán & 90.00 & 8.00 \\
\hline 19 & deltametrina + permetrina & 92.50 & 5.50 \\
\hline 1 & pirimifos metil & 93.75 & 4.25 \\
\hline 4 & paratión metílico & 93.75 & 4.25 \\
\hline 22 & deltametrina + endosulfán & 93.75 & 4.25 \\
\hline 23 & clorpirifos etil + paratión metílico & 93.75 & 4.25 \\
\hline 24 & clorpirifos etil + permetrina & 93.75 & 4.25 \\
\hline 17 & deltametrina + clorpirifos etil & 95.00 & 3.00 \\
\hline 18 & deltametrina + paratión metílico & 95.00 & 3.00 \\
\hline 8 & endosulfán & 96.25 & 1.75 \\
\hline 11 & pirimifos metil + clorpirifos etil & 96.25 & 1.75 \\
\hline 14 & pirimifos metil + clorpirifos metil & 96.25 & 1.75 \\
\hline 15 & pirimifos metil + carbaryl & 96.25 & 1.75 \\
\hline 21 & deltametrina + carbaryl & 96.25 & 1.75 \\
\hline 26 & clorpirifos etil + carbaryl & 96.25 & 1.75 \\
\hline 5 & permetrina & 97.5 & 0.5 \\
\hline 6 & clorpirifos metil & 97.5 & 0.5 \\
\hline 7 & carbaryl & 97.5 & 0.5 \\
\hline 13 & pirimifos metil + permetrina & 97.5 & 0.5 \\
\hline 16 & pirimifos metil + endosulfán & 97.5 & 0.5 \\
\hline 2 & deltametrina & 98.75 & 0 \\
\hline 3 & clorpirifos etil & 98.75 & 0 \\
\hline 9 & cal comercial & 98.75 & 0 \\
\hline 10 & pirimifos metil + deltametrina & 98.75 & 0 \\
\hline 25 & clorpirifos etil + clorpirifos metil & 98.75 & 0 \\
\hline 12 & pirimifos metil + paratión metílico & 100 & 0 \\
\hline 38 & Testigo & 98.75 & 0 \\
\hline
\end{tabular}

No. Trat.=Número de tratamiento, \%GER=Porcentaje de germinación inicial en semillas, \%RED. GERM.=Porcentaje de reducción de la germinación al final de la prueba.

metílico deltametrina + endosulfán. En cuanto a los insecticidas permetrina y carbaryl tuvieron buenos resultados al mezclarse con los demás insecticidas: permetrina + pirimifos metil, permetrina + clorpirifos etil, permetrina + paratión metilico, permetrina + clorpirifos metil, permetrina + carbaryl y permetrina + endosulfán; carbaryl + pirimifos metil, carbaryl + clorpirifos etil, carbaryl + paratión metilico, carbaryl + clorpirifos metil y carbaryl + endosulfán.

\section{Pruebas de germinación.}

En cuanto a los efectos que ocasionó el insecticida sobre la germinación, se tiene que: Los tratamientos evaluados en este estudio no afectan la viabilidad de la semilla; ya que inicialmente se tuvo un $98 \%$ de germinación y después de los seis meses que duro almacenada la semilla, los tratamientos presentaron germinación de un 87.5 a un 98.75 , siendo la reducción en germinación de un $10.50 \%$, la cual se considera aceptable (Cuadro 4). En cuanto a la prueba de germinación, no se aplicó un análisis de varianza, ya que no se establecieron repeticiones, únicamente se junto semilla de las tres repeticiones para hacer un compuesto balanceado.

\section{CONCLUSIONES}

Se identificaron como insecticidas sobresalientes en este estudio: deltametrina, permetrina y carbaryl, las mezclas pirimifos metil + deltametrina, clorpirifos etil + carbaryl, permetrina + carbaryl, pirimifos metil + carbaryl y deltametrina + carbaryl.

La viabilidad de la semilla no fue afectada por los insecticidas, en las dosis aplicadas para cada tratamiento.

\section{LITERATURA CITADA}

AGUILERA P; MA. M. 1991. Validación semicomercial de polvos vegetales y minerales para el combate de Sitophilus zeamais MOTSCH; Prostephanus truncatus HORN. y Rhyzopertha domínica FABR. en el sur y sureste de México. (Tesis Maestría). Colegio de Postgraduados. Montecillo, México. 138 p.

BAÑUELOS M, A. 1992. Depredación de Teretriosoma nigrescens Lewis(Coleoptera: Histeridae) sobre dos biotipos de Prostephanus truncatus Horn (Coleoptera: Bostrichidae) (Tesis Licenciatura) U.A.Ch. Chapingo, México. 69 p.

BURKHOLDER, W. E. 1985. Pheromones for monitoring and control of stored product insects. In: Annual Review of Entomology. Annual Review Inc. California, U.S.A. V. 30:257-272.

COMISIÓN INTERSECRETARIAL PARA EL CONTROL DEL PROCESO Y USO DE PLAGUICIDAS, FERTILIZANTES Y SUSTANCIAS TÓXICAS (CICOPLAFEST). Catálogo Oficial de Plaguicidas 1994. México, D. F. 416 p.

FACIO P; F. 1983. Acondicionamiento de semillas, memorias del curso de actualización de tecnología de semillas. U.A.A.A.N. Saltillo, Coahuila. 87 p.

GARRIDO C; M. G. 1970. Evaluación de tres insecticidas como protectores de granos almacenados contra el ataque de Sitophillus Zeamais (motsch). y Prostephanus truncatus (Horn). (Tesis Maestría). E.N.A. Chapingo, México. 64 p. 
GONZÁLEZ M; M. A. 1988. Diccionario de especialidades agroquímicas. 2a. ed. Ediciones F.L.M. México. 645 p.

LINDBLAD, C.; DRUBEN, L. 1986. Almacenamiento del grano manejo-secado-silos. Control de insectos y roedores. Editorial CONCEPTO S.A. México, D. F. $331 \mathrm{p}$.

MARTÍNEZ, P. P.; VELAZCO, P. H. 1982. Observaciones preliminares sobre la incidencia y daño en maíz por insectos de los granos almacenados en Valles Centrales de Oaxaca: Folia Entomol. Mex. 54: 66

MENSAH, G. W. K.; WHITE, N. D. G. 1984. Laboratory evaluation of malathion-treated sawdust for control of stored -product insects in empty granaries and food warehouses. J. Econ. Entomol. 77 (1):202-206.

RAMAYO L, F. 1983. Tecnología de granos. U.A.Ch. México. 216 p.

RAMÍREZ G; M. 1978. Almacenamiento y conservación de granos y semillas. C.E.C.S.A. México. 300 p.
RAMÍREZ , M. M.; MORENO M., E.; MAC GREGOR L., R.; RAMOS E., J. 1980 Las investigaciones en la Universidad Nacional Autónoma de México sobre protección de productos almacenados. Folia Entomol. Mex. 45: 104 .

REES, D. P. 1991. The effect of Teretriosoma nigrescens Lewis Coleoptera: Histeridae) on three species of storage Bostrichidae infesting shelled maize. J. Stored-0 Prodres. Kent, U. K. U.S.A. 27 (1): 83-86.

SÁNCHEZ A; H. 1987. Actividad de polvos minerales para el combate de Prostephanus truncatus HORN. y Sitophilus zeamais MOTSCHULSKY, en maíz almacenado. (Tesis Maestría). Colegio de Postgraduados. Chapingo, México. 67 p.

WRIGHT, V. F; MILLS, R. B.; WILLCUTTS, B. J. 1987. Methods for culturing stored-grain insects. In: Toward insect resistant maize for the third world. Proceedings of the international symposium on methodologies for developing host plant resistance to maize Insects. CIMMYT. México. pp. 74-83. 